\title{
Synthesis of Chitosan Nanoparticles for Controlled Release of Amiodarone
}

\author{
N. I. BUYUK'1, P. P. ARAYICI, S. DERMAN, Z. MUSTAFAEVA AND S. YUCEL* \\ Department of Bioengineering, Yildiz Technical University, Davutpasa Street, Istanbul, 34220, ${ }^{1}$ Department of Biotechnology, \\ Yeditepe University, Istanbul, Turkey
}

Buyuk et al:: Amiodarone-loaded Chitosan Nanoparticles

\begin{abstract}
This work is based on a natural polymer chitosan used in a nanoparticulate drug delivery system for the controlled release of amiodarone along with $\beta$-cyclodextrin. Amiodarone-loaded chitosan nanoparticles were prepared using the ionic gelation method aided by sonication. Amiodarone loading on chitosan nanoparticles was done under optimum conditions. For particle characterization; zeta-sizer, UV/Vis, Fourier-transform infrared spectroscopy, X-ray powder diffraction, differential scanning calorimeter and scanning electron microscope techniques were used. In vitro drug release studies of amiodaroneloaded chitosan nanoparticles were performed using the dialysis diffusion technique. Drug loading and release values were determined using UV/Vis spectroscopy. Amiodarone encapsulated in nanoparticles was completely released at the end of 14 days. About $38 \%$ was released at the end of day $1,44 \%$ released at the end of day 3,50\% released at the end of day 5 followed slow release. Amiodarone-loaded chitosan nanoparticles could serve as a model for controlled delivery of many antiarrhythmic drugs.
\end{abstract}

Key words: Amiodarone, chitosan nanoparticles, cyclodextrin, drug release, ionic gelation method

The most commonly used natural polymer in drug release systems is chitosan (CS). CS is positively charged compared to other natural polymers and also biocompatible ${ }^{[1]}$. It can leave the system without any side effects. If is nanostructured, it can be used in drug delivery systems. Advantages of nanoparticles (NP) are biocompatibility, biodegradability, low toxicity, control of the release of active agents, production without dangerous organic solvents, cross-linking due to free amine groups in the structure, easy ionic bonding with anion molecules since it is cationic, and mucoadhesive properties $^{[2-4]}$. The preparation method of CSNPs provides a higher affinity for negatively charged biological membranes and specific targeting in vivo. Thanks to its high affinity, it can be used in controlled drug delivery systems ${ }^{[4]}$.

Generally ionic gelation is used for producing CSNPs. The method was first developed in 1997 by Calvo ${ }^{[5]}$. Physical cross-linkers are used because of the potential side effects of chemical cross-linkers in this method. The most extensively used crosslinker for this purpose is sodium tripolyphosphate (TPP), which is a polyanion and makes ionic interactions between CS chains ${ }^{[5]}$. This method eliminates the need for organic solvents for the

*Address for correspondence

E-mail: yuce.sevil@gmail.com

January-February 2020 production of NPs, the reaction can be accomplished in an aqueous solution and this avoids any damage to the activity of the drug since no high energy is needed during NP formation. Using ultrasonic waves to produce NP is one of the most efficient method used in recent times. In sonochemistry general frequency range varies between $20 \mathrm{kHz}-1 \mathrm{MHz}^{[6]}$, with this energy, the formation of aggregates in solution can be broken down, the particle size and also polydispersity index can be reduced ${ }^{[7]}$.

The use of ultrasonic method in nanotechnology has been widely accepted, but the number of studies on CS polymer are very few ${ }^{[8]}$. Tang et al. ${ }^{[8]}$, Saha et al. ${ }^{[9]}$, Rafeeq et al. ${ }^{[10]}$ and Azelea et al. ${ }^{[11]}$ synthesized CSNPs by ionic gelation and then the samples were treated by sonication. After 2014; Gouda et al. ${ }^{[12]}$, Sugita et al. ${ }^{[13]}$, Xu et al. ${ }^{[14]}$ and Chouljenko et al..$^{[15]}$ used only a sonicator device to produce CSNPs. These results

\footnotetext{
This is an open access article distributed under the terms of the Creative Commons Attribution-NonCommercial-ShareAlike 3.0 License, which allows others to remix, tweak, and build upon the work non-commercially, as long as the author is credited and the new creations are licensed under the identical terms
}

Accepted 09 December 2019

Revised 23 September 2019

Received 27 Juy 2019

Indian J Pharm Sci 2020;82(1):131-138 
showed that sonication is helpful in many ways for production of CSNPs.

The production of polymeric NPs is particularly important in the treatment of diseases that require long-term therapy. Today, heart diseases are the most common cause of sudden death in the world. Many of the heart diseases are caused by rhythmic disorders. Antiarrhythmic drugs are widely used to treat rhythmic disorders $^{[16]}$. Amiodarone (AMD) is one of the most prescribed antiarrhythmic drug worldwide that is a class III antiarrhythmic drug ${ }^{[17]}$. Although it has properties in class I, II and IV antiarrhythmic drugs, its main target is the blocking of the $\mathrm{K}$ channel. In addition, AMD is described as a multi-channel blocker because it can also block $\mathrm{Na}$ and $\mathrm{Ca}$ channels. Blocking the $\mathrm{K}$ channel prolongs atrial and ventricular repolarization time ${ }^{[18]}$. It has a slowing effect on heart rate.

NPs, which have been widely prepared for controlled drug delivery has advantages such as, controlled release and ease of entry into the cell due to the fact that they are nano-sized. Natural polymers are desired drug release materials because of having the advantages of NPs.

This work is based on employing a natural polymer, CS due to its advantages, in drug delivery systems. In this study, an attempt was made to load AMD, one of the most commonly used antiarrhythmic drug, in biocompatible and biodegradable CSNPs for use in long-term treatment of heart diseases. Although antiarrhythmic drug-loaded CSNP studies are available in the literature ${ }^{[19-21]}$, the number of these studies is quite low due to the hydrophobicity and difficult encapsulation of the active substances. In these studies, organic solvents were generally used to dessolve such lipophilic drugs and this could cause carcinogenic or neurotoxic effects.

Since AMD is also hydrophobic, $\beta$-cyclodextrin $(\beta-C D)$, which has been extensively used to increase the water solubility of hydrophobic molecules ${ }^{[22-29]}$, was added to enhance AMD solubility in water and allow it to be loaded into the CSNP. A Zeta-Sizer, UV/Vis spectrometry, Fourier-transform infrared (FTIR), X-ray diffraction (XRD), differential scanning calorimetry (DSC) and scanning electron microscopy (SEM) analytical techniques were used to characterize the NPs produced and controlled release of AMD from these NPs was determined in phosphate buffered saline (PBS).

\section{MATERIALS AND METHODS}

Chitosan (deacetylation degree- 75-85 \%, medium molecular weight, product code: 448877), AMD hydrochloride (purity- $98 \%$ purity, molecular weight- 681.77, product code- A8423)and sodium tripolyphosphate (molecular weight- 367.86, product code: 72061) were purchased from Sigma-Aldrich; $\beta$-cyclodextrin (molecular weight- 1134.98, product code- 1154569) was purchased from USP; was purchased from Sigma-Aldrich. All other chemicals used were of analytical grade.

\section{AMD solubility studies:}

AMD solubility studies were performed according the method of Higuchi et al. ${ }^{[30]}$. Study was performed in two steps; in the first step, $20 \mathrm{mg} \beta$-CD was added to 10 $\mathrm{ml}$ pure water containing $1,3,5,10$ and $20 \mathrm{mg}$ AMD, respectively. In the second step, AMD was added to $10 \mathrm{ml}$ pure water containing 20,25, 30, 35 and $40 \mathrm{mg}$ $\beta-C D$, respectively. These were stirred on a magnetic stirrer at room temperature for $24 \mathrm{~h}$. The solutions were filtered through $0.45 \mu \mathrm{m}$ syringe filters. The filtered solutions were analyzed by the UV spectrophotometer (Scinco, S-3100) at $242 \mathrm{~nm}^{[31]}$.

\section{Preparation of the AMD-loaded CSNPs:}

CSNPs were synthesized by the enhanced method of ionic gelation, which was developed by $\mathrm{Calvo}^{[5]}$ and then advanced using the ultrasonicator ${ }^{[8,13,32]}$. The AMD- $\beta$-CD solution was added to the CS solution at a concentration of $4 \mathrm{mg} / \mathrm{ml}(\mathrm{w} / \mathrm{v})$ and filtered through a $0.45 \mu \mathrm{m}$ syringe filter. The solution was stirred on a magnetic stirrer for $30 \mathrm{~min}$ to provide an interaction between CS and AMD. The TPP solution at a concentration of $2 \mathrm{mg} / \mathrm{ml} \mathrm{(w/v)} \mathrm{was} \mathrm{filtered} \mathrm{through} \mathrm{a}$ $0.20 \mu \mathrm{m}$ syringe filter. Then, at room temperature, the ultrasonicator set for $5 \mathrm{~min}$ at $50 \mathrm{~W}$ with $30-10$ pulse. The AMD-loaded NPs were synthesized by the mass ratio of 5:1 (CS:TPP). The synthesis conditions used in the study and given formulation codes (FC) are presented in the Table 1.

\section{Particle size and surface charge determination:}

A Zetasizer Nano ZS (Model ZEN0040, Malvern Instruments) was used for characterization. Particle size, zeta potential and polydispersity index (PDI) of blank and AMD-loaded NPs were determined by photon correlation spectroscopy. Blank and AMD-loaded CSNPs were prepared under the specified conditions. Dynamic light scattering (DLS) technique was used 
TABLE 1: DIFFERENT SYNTHESIS CONDITIONS USED IN THE STUDY

\begin{tabular}{lllcc}
\hline FC & $\begin{array}{l}\text { CS content } \\
(\mathrm{mg} / \mathrm{ml})\end{array}$ & $\begin{array}{l}\text { TPP content } \\
(\mathrm{mg} / \mathrm{ml})\end{array}$ & $\begin{array}{c}\beta-C D \text { content } \\
(\mathrm{mg} / \mathrm{ml})\end{array}$ & $\begin{array}{c}\text { AMD } \\
\text { content } \\
(\mathrm{mg} / \mathrm{ml})\end{array}$ \\
\hline NP1 & 4 & 2 & 3.0 & - \\
NP2 & 4 & 2 & 3.5 & - \\
NP3 & 4 & 2 & 3.0 & 0.5 \\
NP4 & 4 & 2 & 3.5 & 0.5 \\
\hline
\end{tabular}

to calculate the average size of CSNPs. About $500 \mu 1$ of NP solution was loaded directly into the disposable cuvette of the zetasizer by placing it in the device ${ }^{[33]}$.

\section{Morphology and FTIR analysis:}

SEM (Zeiss, EVOLS10, Japan) was used to determine the morphological characteristics of NPs. For SEM analysis, one drop of freshly prepared NPs dropped onto the carbon band and the liquid part is evaporated at room temperature. The surface was coated with a gold-palladium complex under vacuum and then examined ${ }^{[34]}$. Molecular characterization of blank and AMD-loaded CSNPs was performed on a IR-Prestige 21 FTIR spectrophotometer (Shimadzu, Japan). The device was set with resolution of $4 \mathrm{~cm}^{-1}$ and with frequency range of $650-4000 \mathrm{~cm}^{-1}$.

\section{XRD and DSC analyses:}

The crystal structure of the synthesized NPs was measured by XRD. The XRD measurements were performed on an X'Pert PRO (PANalytical BV, Netherlands). X-ray diffraction patterns were recorded using $\mathrm{Cu} \mathrm{K \alpha}$ radiation, operated at $45 \mathrm{kV}$ voltage and $40 \mathrm{~mA}$ current. The samples were recorded $0.025^{\circ}$ step resolution, in the $2 \theta$ ranges from $10^{\circ}$ to $60^{\circ}$ for the AMD, blank and loaded NPs. DSC analysis was performed using DSC131 (Setaram, KEP Technologies, France) to learn NPs heat capacity changed by temperature. The phase behavior of CS, TPP, AMD, CD, blank and AMD loaded NPs was recorded. About $6 \mathrm{mg}$ of samples for each were accurately weighed and sealed in an aluminum pan. Thermogram was recorded in a range of $50-350^{\circ}$ with heating rate of $10^{\circ} / \mathrm{min}$.

\section{Evaluation of AMD-loaded NP reaction yield:}

The reaction yield (RY) analysis of the AMD-loaded CSNPs was determined by gravimetric method. The CSNP suspension was centrifuged at $+4^{\circ}, 13000 \mathrm{rpm}$ for $30 \mathrm{~min}$ in a high-speed refrigerated centrifuge (Hitachi) with a 50-ml falcon tube. The supernatant was removed, the pellet was washed several times and the remaining pellet suspensions were lyophilized. using an Alpha 1-2 LDplus lyophilizer. Fully dried NPs were weighed on a precision scale. The yield was calculated using the following Eqn., yield (\%) = (weight of NPs)/ (total weight of solids) $\times 100$.

\section{Evaluation of encapsulation efficiency and loading capacity:}

The encapsulation efficiency (EE) and loading capacity (LC) of the AMD-loaded CSNPs were measured using the supernatant collected after centrifugation of NPs. The absorbance value of the obtained supernatants was measured at $242 \mathrm{~nm}^{[35]}$. AMD concentration was determined using the $\varepsilon$, molar absorption coefficient. The $\mathrm{EE}^{[36]}$ and $\mathrm{LC}^{[37]}$ were calculated using the Eqns. 2 and $3, \mathrm{EE}(\%)=($ total amiodarone amount-amiodarone amount in supernatant $) /($ total amiodarone amount $) \times 100$; LC $(\%)=$ (total amiodarone amount-amiodarone amount in supernatant $) /($ weight of nanoparticles $) \times 100$.

\section{In vitro release of AMD from CSNPs:}

Determination of in vitro release was performed by placing the dried AMD-loaded CSNPs in a beaker containing $5 \mathrm{ml}$ of PBS and $0.01 \%$ sodium azide solution. The sample was sonicated and placed on a dialysis membrane (MWCO: 12.000 Da, D9777, Dialysis tubing cellulose membrane, Sigma). The dialysis membrane was placed in a beaker contains 50 $\mathrm{ml}$ of PBS. The temperature of the shaking water bath was adjusted to $37^{\circ}$ and agitation was started at 100 $\mathrm{rpm}$. Samples were withdrawn at specific time intervals and same volume of fresh PBS solution was replaced each time ${ }^{[38]}$. The AMD concentration was determined on a UV/Vis spectrophotometer at $242 \mathrm{~nm}^{[35]}$.

\section{RESULTS AND DISCUSSION}

The phase solubility profiles for the complex formation between AMD and $\beta-C D$ was shown in fig. 1. The

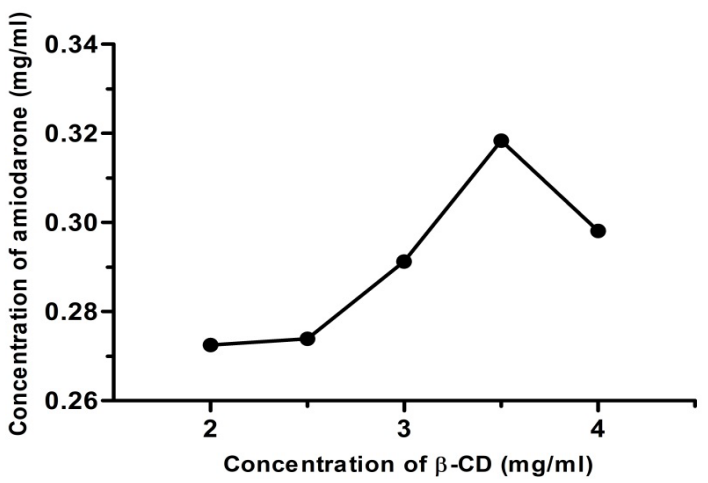

Fig. 1: Phase solubility of amiodarone with $\beta$-cyclodextrin Phase solubility plot of amiodarone with $\beta$-cyclodextrin in distilled water at $25 \pm 0.5^{\circ}$ 
plot of the AMD $\beta$-CD solubility study indicated a characteristic Bs type phase solubility diagram ${ }^{[39]}$. B type curves are seen in weakly soluble substances in water. The Bs type is found for materials with limited water solubility. It appears that AMD is dissolved in water in limited quantities because of the hydrophobic character ${ }^{[16]}$. Only $30 \%$ of the AMD was dissolved in water. It was decided to use solutions containing 30 and $35 \mathrm{mg}$ of $\beta-\mathrm{CD}$, which were the best conditions to dissolve. These two conditions had been used in all experiments in this study.

Particle size and zeta potential measurements were performed on a zetasizer. Dimensional analysis was performed and recorded. The results were presented in Table 2. The mean Z-avg sizes of blank NPs ranged from $172.5 \pm 8.19$ to $228.3 \pm 9.73 \mathrm{~nm}$ while AMDloaded NPs ranged from $296.8 \pm 4.14$ to $372.8 \pm 11.53$ $\mathrm{nm}$. The intensity magnitudes of blank NPs varied from $216.4 \pm 15.70$ to $363.7 \pm 40.21 \mathrm{~nm}$ while intensity magnitudes of AMD-loaded NPs varied between $377.4 \pm 31.44$ to $484.5 \pm 45.33 \mathrm{~nm}$. The size distribution patterns were presented in fig. 2. It appeared that the size increased when AMD was loaded to NPs. Similarly Elzatahry et al. ${ }^{[40]}$ reported that blank NPs prepared with a mass ration of 4:1 (CS:TPP), the blank NP diameter was $300 \mathrm{~nm}$, which increased to $450 \mathrm{~nm}$ after metronidazole was loaded. Ye et al. ${ }^{\left[{ }^{[6]} \text { synthesized }\right.}$ blank NPs with a size of 238.5 to $391.2 \mathrm{~nm}$ and when these NPs were loaded with paclitaxel/dimethyl- $\beta-C D$, larger particles with diameters ranging from 440.2 to $521.4 \mathrm{~nm}$ were formed. In the present study also it was observed that the particle size increased with drug loading as reported in literature ${ }^{[28,41-44]}$.

Zeta potential values were found in the range of 26-28 $\mathrm{mV}$ in blank NPs and 29-30 mV in AMD-loaded NPs. There was no significant difference between the zeta potentials of blank and AMD-loaded CSNPs (Table 2). Since the zeta potential values are around $30 \mathrm{mV}$, the solution tends to be stable ${ }^{[45]}$.

The particle morphology characteristics of the AMDloaded CSNPs were investigated using SEM. The SEM

TABLE 2: PHYSICOCHEMICAL PROPERTIES OF BLANK AND AMIODARONE-LOADED CSNPS

\begin{tabular}{lccccccc}
\hline FC & Z-avg $(\mathrm{nm})$ & PDI & Intensity $(\mathrm{nm})$ & Zeta Potential $(\mathrm{mV})$ & RY $(\%)$ & EE $(\%)$ & LC (\%) \\
\hline NP1 & $172.5 \pm 8.2$ & $0.39 \pm 0.048$ & $216.4 \pm 15.7$ & +27.2 & 11.9 & - & - \\
NP2 & $228.3 \pm 9.7$ & $0.48 \pm 0.033$ & $363.7 \pm 40.2$ & +26.0 & 11.8 & - & - \\
NP3 & $296.8 \pm 4.1$ & $0.41 \pm 0.023$ & $377.4 \pm 31.4$ & +29.4 & 13.3 & 33.4 & 8.6 \\
NP4 & $372.8 \pm 11.53$ & $0.44 \pm 0.036$ & $484.5 \pm 45.33$ & +29.7 & 14.2 & 35.6 & 8.8 \\
\hline
\end{tabular}
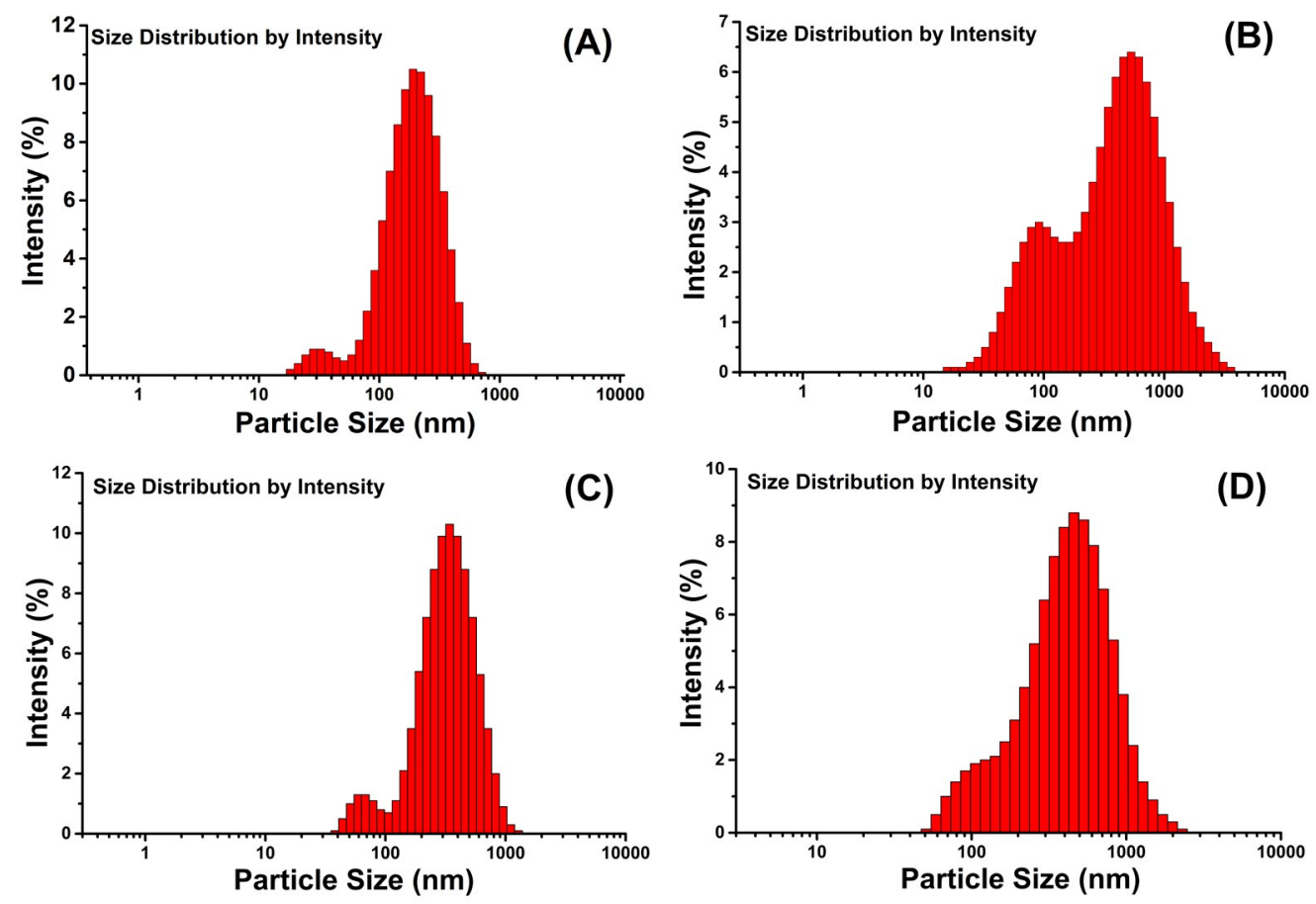

Fig. 2: CSNP particle size distribution Particle size distribution of the Chitosan Nanoparticles (CANP) (A) NP1, (B) NP2, (C) NP3 and (D) NP4 


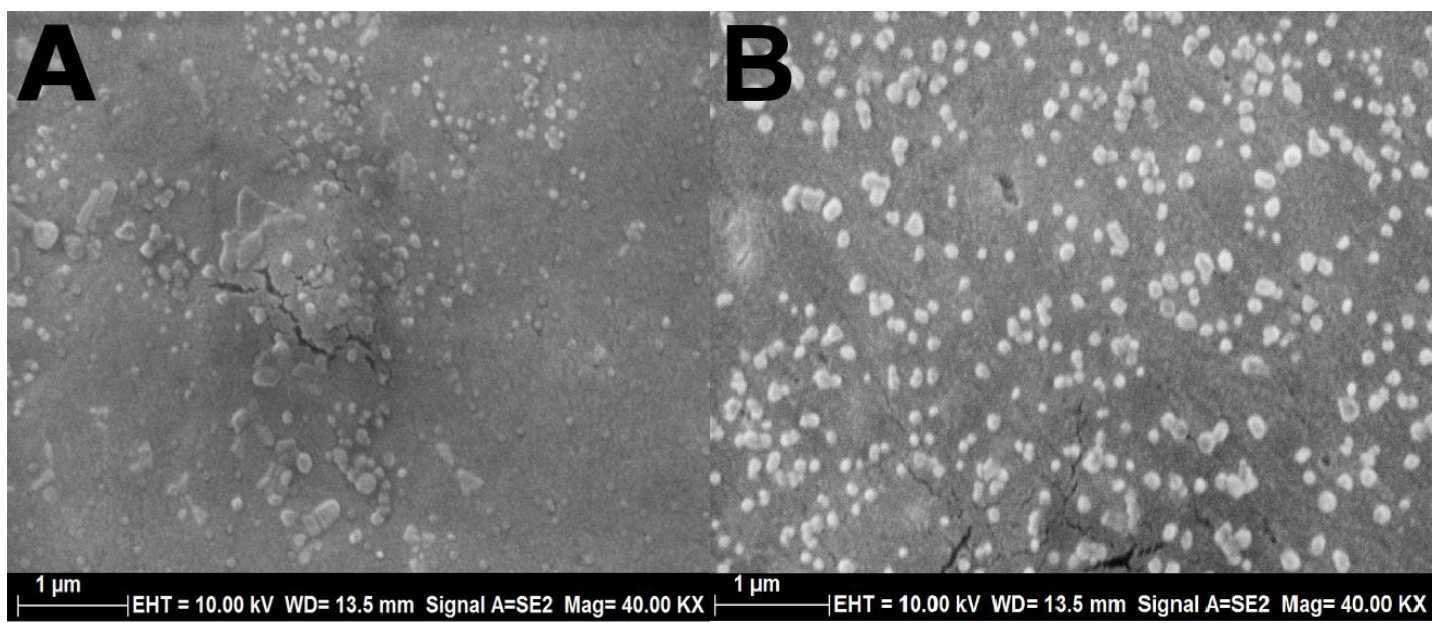

Fig. 3: Scanning electron pictomicrographs of AMD-loaded CSNPs

Scanning electron pictomicrographs of amiodarone-loaded chitosan nanoparticles (AMD-loaded CSNP) (A) NP3, and (B) NP4, produced by sonication

image of AMD-loaded CSNPs was presented in the fig. 3. The results showed that AMD-loaded CSNPs were spherical in shape and had smooth surface. NP4 was bigger than the NP3 because of the increased loading content. The particle size results obtained using DLS and SEM image showed consistency.

The FTIR spectra of CS, AMD, $\beta-C D$, blank and AMD-loaded CSNPs are shown in fig. 4. Characteristic absorbance peaks of CS with medium molecular weight are 3396 and $3284 \mathrm{~cm}^{-1}$, which are due to $-\mathrm{NH}$ and -OH stretching in CS. These peaks may also be due to hydrogen bonds for the molecule. The 2921 and $2850 \mathrm{~cm}^{-1}$ peaks are $-\mathrm{CH}$ symmetric and asymmetric stresses, respectively. These bands are characteristic for polysaccharides and are also found in other polysaccharides. As residues of $\mathrm{N}$-acetyl groups of $\mathrm{CS}$ structure peaks are; $1640 \mathrm{~cm}^{-1}(\mathrm{C}=\mathrm{O}$ stretching $), 1300$ $\mathrm{cm}^{-1}$ (-CN stretching), $1545 \mathrm{~cm}^{-1}$ (-NH stretching), 1004 $\mathrm{cm}^{-1}$ (C-O stretching) ${ }^{[46]}$. In the FTIR spectroscopy, the AMD molecule gives the characteristic peaks at 1620 , $1557,1170,998$ and $754 \mathrm{~cm}^{-1}$. Stress vibrations of the group - $\mathrm{C}=\mathrm{O}$ carbonyl appear at $1620 \mathrm{~cm}^{-1}$; the peaks at 1557 and $1446 \mathrm{~cm}^{-1}$ represent aromatic $\mathrm{C}=\mathrm{C}$ stretching vibrations. Aliphatic $\mathrm{CH}$ stretching is presented at symmetrical and asymmetric stretching of 2931 and $2855 \mathrm{~cm}^{-1}$, aromatic $\mathrm{CH}$ stretching at $2968 \mathrm{~cm}^{-1}$, aliphatic $\mathrm{CH}_{3}$ symmetric deformation at $1381 \mathrm{~cm}^{-1}, \mathrm{C}=\mathrm{O}$ stretching at $1620 \mathrm{~cm}^{-1[35]}$. The peaks originating from $-\mathrm{NH}$ and $-\mathrm{OH}$ stretching in the CSNPs were found to expand to 3427,3276 and $3157 \mathrm{~cm}^{-1}$ peaks, respectively, for AMD-loaded samples. This situation is thought to be due to the expansion of $\mathrm{H}$ bonds ${ }^{[47]}$. It oberved that $1640 \mathrm{~cm}^{-1}$ peak in the CS is protected in NPs however $1545 \mathrm{~cm}^{-1}$ absorbance peak shifted to $1552 \mathrm{~cm}^{-1}$. This

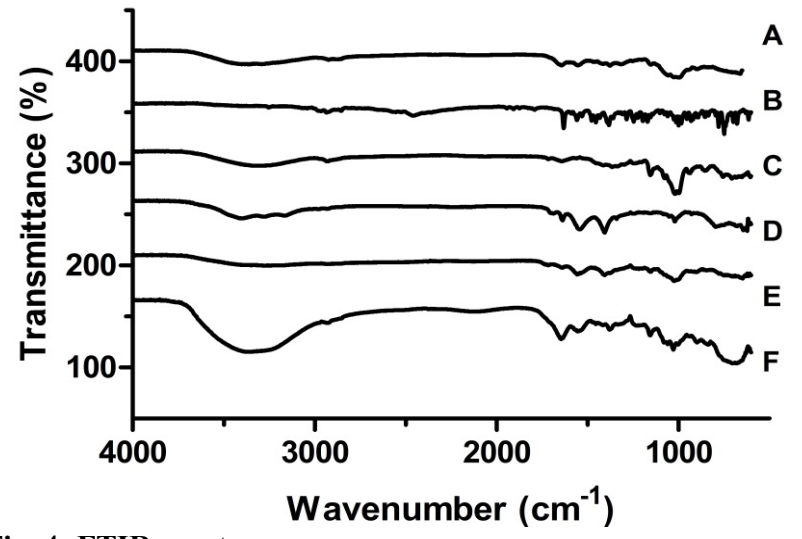

Fig. 4: FTIR spectra

(A) Chitosan, (B) amiadarone, (C) $\beta$-CD, (D) blank chitosan nanoparticles, (E) amiodarone-loaded chitosan nanoparticle NP3, (F) amiodarone-loaded chitosan nanoparticle NP4

is thought to be due to the cross-linking of - $\mathrm{NH}$ bonds with TPP. A new peak formation originating from $\mathrm{P}=\mathrm{O}$ bond was observed at $1209 \mathrm{~cm}^{-1[48]}$. This is due to the formation of bonds between the amino groups present in the CS and the phosphate groups present in TPP. The characteristic peaks of AMD disappear after NP production. This situation proves that the drug is effectively encapsulated ${ }^{[49]}$.

The physical state of AMD, blank and loaded NPs were subjected to XRD analysis. The diffraction spectrums of samples were presented in fig. 5. While AMD shows significant peaks at $21.46^{\circ}, 23.47^{\circ}$, $27.17^{\circ}$ due to its crystal structure, it was seen that the intensity peaks in blank and loaded nanoparticles is diminished. This proved that AMD was loaded into chitosan nanoparticles quite successfully. At the same time, AMD in nanoparticles lost its crystallinity, which is a less soluble form to its amorphous form. Studies reported in literature support this finding ${ }^{[50]}$. The 
magnitude of the peaks obtained as a result of XRD was associated with the crystalline form of the material. According to XRD results obtained from nanoparticles, these peaks seem to be quite low, which was thought to be caused by the loss of the crystal structure of chitosan as a result of cross-linking with $\operatorname{TPP}^{[22]}$.

The phase transition curves of CS, TPP, AMD, $\beta-C D$, blank and empty NPs are presented in fig. 6. In CS thermogram two peaks exhibited: an endothermic peak at $120.27^{\circ}$ due to loss of water and exothermic peak at $310.72^{\circ}$ due to decomposition of amine groups ${ }^{[51]}$. AMD showed a single sharp endothermic peak at $172.77^{\circ}$ according to the melting temperature of the drug. Thermogram of CD showed two endothermic peaks at $140.67^{\circ}$ and $279.06^{\circ}$. In addition, thermogram of TPP showed two endothermic peaks at $121.05^{\circ}$ and $206.98^{\circ}$ corresponding to melting point of crystalline region. Blank and loaded NPs had an endothermic peak at $107.91^{\circ}$ and $97.59^{\circ}$ and also another endotherm peak at 275.63 and $277.6^{\circ}$, respectively. In NPs characteristics

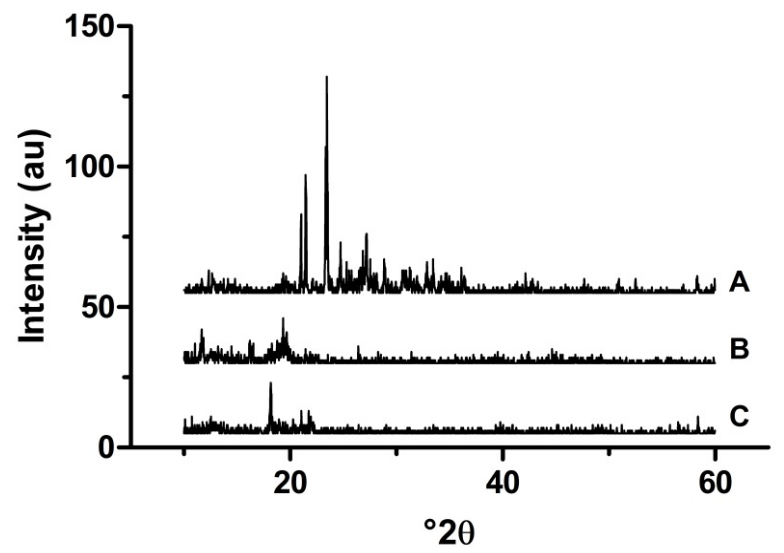

Fig. 5: XRD spectra

(A) Amiodarone, (B) blank chitosan nanoparticles, (C) amiodarone-loaded chitosan nanoparticles

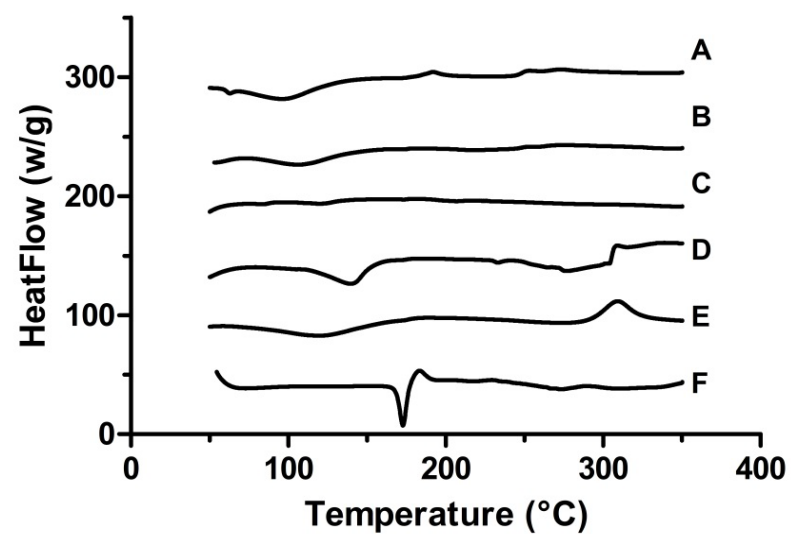

Fig. 6: DSC spectra

(A) Amiodarone-loaded chitosan nanoparticles, (B) blank chitosan nanoparticles, (C) TPP, (D) $\beta$-CD, (E) chitosan, (F) amiodarone peaks of CS and $\beta-C D$ were joint and could observed. There were no endothermic peak for AMD in loaded NPs, which showed that AMD had been incorporated with $\beta-C D^{[50]}$. Similar results were obtained in the literature $^{[52]}$.

The RY of blank and AMD-loaded CSNPs varied between 11-15\% (Table 2). AMD-loaded NPs showed low RY values. No significant difference was observed in RYs between NPs loaded with different amounts of CD. RY was higher in NPs loaded with active substance than blank NPs, which was thought to be due to the fact that $\mathrm{CD}$, which capsulated AMD, forms a stronger bond with CS to produce NPs. In the study of Trapani et al. loading materials with low water solubility into CSNP, the RY remained in the range of 8-13\% which was an acceptable range for hydrophobic materials ${ }^{[25]}$.

The EE of AMD-loaded CSNPs varied between 33 and $36 \%$. The EE results of this study showed similar trends to what was reported in the literature ${ }^{[26]}$. The LC of AMD-loaded CSNPs varied between 8-9 \% (Table 2). Although the results obtained in this study are similar to the previous studies ${ }^{[25]}$, the LC in this study is slightly more effective ${ }^{[42]}$. It was found that increasing the amount of CD in NPs, which is used to increase the solubility of AMD, also increased the EE and LC of CSNPs, but this increase is not significant. Although it is convenient for such molecules to increase the amount of $\mathrm{CD}$ used for loading hydrophobic materials to chitosan, it is seen that EE and LC are lower compared to other studies performed for loading hydrophilic materials ${ }^{[31,36,44]}$.

Release studies on AMD-loaded CSNPs were carried out in triplicate. Two different doses of drug-loaded NP were used to study release. The study was continued for $20 \mathrm{~d}$. Concentration calculations were made with UV measurements taken at specific time intervals, these values were converted to $\mathrm{mg}$, and fig. 7 was drawn to determine to find the drug release percentage with time. It appears that the NPs which contained $5 \mathrm{mg}$ AMD and $30 \mathrm{mg} \beta-C D$ release all of the AMD it's include at the end of the release study. It took $14 \mathrm{~d}$ for the CSNPs to release $100 \%$ of the drug. About $32 \%$ of the active substance was released within the first $5 \mathrm{~h}$, followed by a slow release. After $14 \mathrm{~d}$, the work was continued without interruption and was shown to be stable. It can be seen that the release of all of AMD in NP4 could not be achieved. It was observed that the CSNP, which released $22 \%$ of AMD loaded in the first $4 \mathrm{~h}$, slowed down the process to released up to $40 \%$ of AMD. This difference between the two NPs produced is thought 


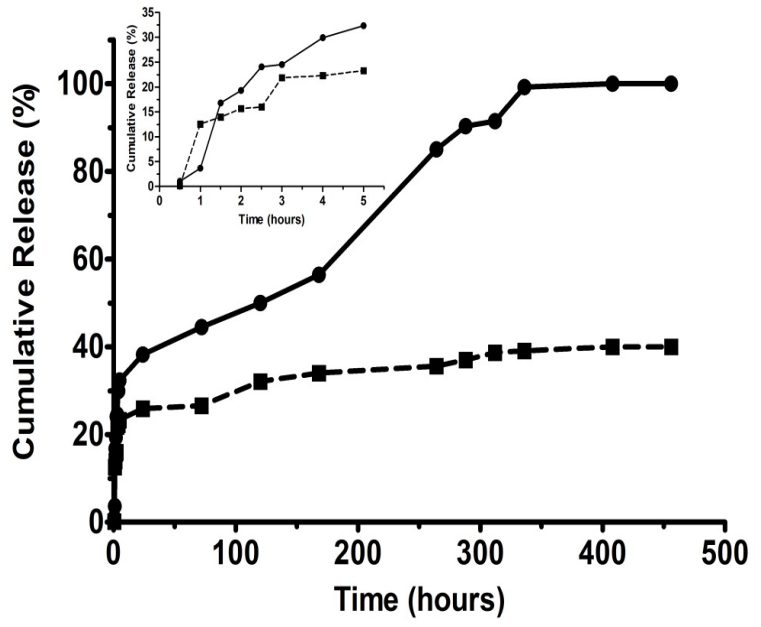

Fig. 7: In vitro drug release from amiodarone-loaded CSNPs In vitro drug release study of amiodarone-loaded chitosan nanoparticles NP3 (-॰-) and NP4 (-ロ-)

to be caused by the change in the amount of $\mathrm{CD}$ it contains. The $\mathrm{CD}$ in the solution provided a tighter binding to the NP, resulting in slowing of release. Recent studies showed that adding CD improved the LC despite slowing down drug release $\mathrm{e}^{[53,54]}$.

In summary, AMD was loaded to CSNPs successfully. Solubility studies were performed with $\beta$-CD due to the hydrophobic nature of AMD. AMD: $\beta-C D$ (1:7) mass ratio was the optimal combination. In the Zetasizer results, it was observed that the NPs were of the optimal size and had monodispersed particle distribution. SEM images showed spherical structure. The fact that the encapsulation of AMD was successful was obtained from the FTIR, DSC and XRD results. DSC and XRD analysis indicated that physical state of AMD transformed into more amorphous state. AMDloaded NPs had a RY of 11-15\%, an EE of 33-36 \% and a LC of 8-9 \% in this study. In in vitro release studies, all of the AMD was released after $14 \mathrm{~d}, 38 \%$ at the end of day $1,44 \%$ at the end of day 3 , and $50 \%$ at the end of day 5. It is thought that NPs produced in this study could be used for long-term treatment with AMD and also could serve as a model for controlled delivery of many antiarrhythmic drugs.

\section{REFERENCES}

1. Yao $\mathrm{KD}$, Yin $\mathrm{YJ}, \mathrm{Xu} \mathrm{MX}$, Wang YF. Investigation of $\mathrm{pH}-$ sensitive drug delivery system of chitosan/gelatin hybrid polymer netw. Polym Int 1995;38:77-82.

2. Peniche C, Argüelles-Monal W, Goycoolea FM. Chitin and Chitosan: Major Sources, Properties and Applications. In: van den Broek LAM, Boeriu CG, Stevens CV, editors. Monomers, polymers and composites from renewable resources. Amsterdam: Elsevier; 2008. p. 517-42.

3. Gan Q, Wang T. Chitosan nanoparticle as protein delivery carrier-Systematic examination of fabrication conditions for efficient loading and release. Colloids Surfaces B Biointerfaces 2007;59(1):24-34.

4. Perera UMSP, Rajapakse N. Seafood Processing By-Products. In: Se-Kwon K, editors. Chitosan Nanoparticles: Preparation, Characterization, and Applications. New York: Springer; 2013. P. 371-87.

5. Calvo P, Lopez CR, Vila-Jato JL, Alonso MJ. Novel Hydrophilic Chitosan - Polyethylene Oxide Nanoparticles as Protein Carriers. J Appl Polym Sci 1997;63:125-32.

6. Schroeder A, Kost J, Barenholz Y. Ultrasound, Liposomes, and Drug Delivery: Principles for using Ultrasound to Control the release of Drugs from Liposomes. Chem Phys Lipids 2009;162:1-16.

7. Tsai M, Bai S, Chen R. Cavitation effects versus stretch effects resulted in different size and polydispersity of ionotropic gelation chitosan-sodium tripolyphosphate nanoparticle. Carbohydr Polym 2008;71:448-57.

8. Tang ESKSK, Huang M, Lim LYY. Ultrasonication of chitosan and chitosan nanoparticles. Int J Pharm 2003;265(1-2):103-14.

9. Saha P, Goyal AK, Rath G. Formulation and evaluation of chitosan-based ampicillin trihydrate nanoparticles. Trop J Pharm Res 2010;9(5):483-8.

10. Rafeeq MP, Junise V, Saraswathi R, Krishnan P. Development and characterization of chitosan nanoparticles loaded with isoniazid for the treatment of Tuberculosis. Res J Pharm Biol Chem Sci 2010;1(4):383-90.

11. Azalea DR, Mohambed M, Joji S, Sankar CBM. Design and Evaluation of Chitosan Nanoparticles as Novel Drug Carriers for the Delivery of Donepezil. Iran J Pharm Sci 2012;8(3):15564.

12. Gouda M, Elayaan U, Youssef MM. Synthesis and Biological Activity of Drug Delivery System Based on Chitosan Nanocapsules. Synth Biol Act Drug Deliv Syst Based Chitosan Nanocapsules Adv Nanoparticles 2014;3(3):148-58.

13. Sugita $P$, Ambarsari L, Lidiniyah. Optimization of ketoprofenloaded chitosan nanoparticle ultrasonic process. Procedia Chem 2015;16:673-80.

14. Xu F, Zhao T, Wang S, Wang H, Cui X. Preparation of magnetic and $\mathrm{pH}$-responsive chitosan microcapsules via sonochemical method. J Microencapsul 2016;33(2):191-8.

15. Chouljenko A, Chotiko A, Solval MJ, Solval K, Sathivel S. Chitosan Nanoparticle Penetration into Shrimp Muscle and its Effects on the Microbial Quality. Food Bioprocess Technol 2017; 10(1):186-98.

16. Gill J, Heel RC, Fitton A. Amiodarone. An overview of its pharmacological properties, and review of its therapeutic use in cardiac arrhythmias. Drugs 1992;43(1):69-110.

17. Marcus FI, Fontaine GH, Frank R, Grosgogeat Y. Amiodarone: the experience of the past decade. Am Heart J 1981;101:48093.

18. Vassallo P, Trohman RG. Prescribing Amiodarone. JAMA 2007;298(11):1312-22.

19. Patil R, Pande V, Sonawane R. Nano and microparticulate chitosan based system for formulation of carvedilol rapid melt tablet. Adv Pharm Bull 2015;5(2):169-79.

20. Khan M saleh, Gowda DV, Shivakumar HG. Porous nanoparticles of metoprolol tartrate produced by spray-drying: Development, characterization and in vitro evaluation. Acta Pharm 2012;62(3):383-94.

21. Duangjit S, Kraisit P, Luangtana-Anan M. An investigation of propranolol-loaded chitosan nanoparticles for transmucosal delivery: Physical characterization. Thai J Pharm Sci 2016;40:25-8. 
22. Jingou J, Shilei H, Weiqi L, Danjun W, Tengfei W, Yi X. Preparation, characterization of hydrophilic and hydrophobic drug in combine loaded chitosan/cyclodextrin nanoparticles and in vitro release study. Colloids Surfaces B Biointerfaces 2011;83(1):103-7.

23. Ji J, Hao S, Liu W. Preparation and evaluation of O-carboxymethyl chitosan/cyclodextrin nanoparticles as hydrophobic drug delivery carriers. Polym Bull 2011;67(7):1201-13.

24. Vyas A, Saraf S, Saraf S. Encapsulation of cyclodextrin complexed simvastatin in chitosan nanocarriers: A novel technique for oral delivery. J Incl Phenom Macrocycl Chem 2010;66(3):251-9.

25. Trapani A, Sitterberg J, Bakowsky U, Kissel T. The potential of glycol chitosan nanoparticles as carrier for low water soluble drugs. Int J Pharm 2009;375(1-2):97-106.

26. Ye YJ, Wang Y, Lou KY, Chen YZ, Chen R, Gao F. The preparation, characterization, and pharmacokinetic studies of chitosan nanoparticles loaded with paclitaxel/dimethyl$\beta$-cyclodextrin inclusion complexes. Int $\mathrm{J}$ Nanomedicine 2015;10:4309-19.

27. Jingou J, Jingfen Z, Shilei H, Danjun WU, Li L, Yi X. Preparation, Characterization of Hydrophobic Drug in Combine Loaded Chitosan / Cyclodextrin / Trisodium Citrate Nanoparticles and in vitro Release Study. Chem Res Chin Univ 2012;28(1):166-70.

28. Gadade DD, Pekamwar S. Cyclodextrin Based Nanoparticles for Drug Delivery and Theranostics. Adv Pharm Bull 2019;19:28.

29. Menezes PDP, Andrade TA, Frank LA, de Souza EPBSS, Trindade GDGG, Trindade IAS, et al. Advances of nanosystems containing cyclodextrins and their applications in pharmaceuticals. Int J Pharm 2019;559:312-28.

30. Higuchi T, Connors KA. Phase-solubility techniques. Adv Anal Chem Instrum 1965;4:117-212.

31. Lu L, Shao X, Jiao Y, Zhou C. Synthesis of chitosan- graft - $\beta$-cyclodextrin for improving the loading and release of doxorubicin in the nanopaticles. J Appl Polym Sci 2014;131(21):1-7.

32. Li J, Huang Q. Rheological properties of chitosantripolyphosphate complexes: From suspensions to microgels. Carbohydr Polym 2012;87(2):1670-7.

33. Mertz MW. Characterization of Novel Chitosan/Polyelectrolyte Nanoparticles, Biomedical Engineering Undergraduate Honors Theses [dissertation]. Fayetteville, AR: University of Arkansas; 2015.

34. Arasoglu T, Derman S, Mansuroglu B, Yelkenci G, Kocyigit B. Synthesis, Characterization and Antibacterial Activity of Juglone Encapsulated PLGA Nanoparticles. J Appl Microbiol 2017;123:1407-19.

35. Bonati M, Gaspari F, D'Aranno V, Benfenati E, Neyroz P, Galletti F. Physicochemical and analytical characteristics of amiodarone. J Pharm Sci 1984;73(6):829-31.

36. Dounighi M, Eskandari R, Zolfagharian H, Mohammad M. Preparation and in vitro characterization of chitosan nanoparticles containing Mesobuthus eupeus scorpion venom as an antigen delivery system. J Venom Anim Toxins Incl Trop Dis 2012;18(1):44-52.

37. Liu Y, Sun Y, Li YL, Xu SC, Xu YX. Characteration of Protein Loaded Chitosan Nanoparticles at Different $\mathrm{pH}$ Values. Adv Mater Res 2011;5:284-286, 950-3.
38. Arasoglu T, Derman S, Mansuroglu B. Comparative evaluation of antibacterial activity of caffeic acid phenethyl ester and PLGA nanoparticle formulation by different methods. Nanotechnology 2015;27:1-12.

39. Loftsson T, Brewster ME, Ru RR. Remington: The Science and Practice of Pharmacy Sample Chapter. In: Troy D, Beringe P, editors. Complex Formation. Philadelphia, Pennsylvania, United States: Lippincott Williams\& Wilkins; 2006. p. 681-92.

40. Elzatahry AA, Eldin MSM. Preparation and characterization of metronidazole-loaded chitosan nanoparticles for drug delivery application. Polym Adv Technol 2008;19(12):1787-91.

41. Hou X, Zhang W, He M, Lu Y, Lou K, Gao F. Preparation and characterization of $\beta$-cyclodextrin grafted $\mathrm{N}$-maleoyl chitosan nanoparticles for drug delivery. Asian J Pharm Sci 2017;12:558-68.

42. Tang P, Sun Q, Zhao L, Pu H, Yang H, Zhang S. Mesalazine/ hydroxypropyl- $\beta$-cyclodextrin/chitosan nanoparticles with sustained release and enhanced anti-inflammation activity. Carbohydr Polym 2018;198:418-25.

43. Matshetshe KI, Parani S, Manki SM, Oluwafemi OS. Preparation, characterization and in vitro release study of $\beta$ cyclodextrin/ chitosan nanoparticles loaded Cinnamomum zeylanicum essential oil. Int J Biol Macromol 2018;118:67682.

44. He M, Zhong C, Hu H, Jin Y, Chen Y, Lou K. Cyclodextrin/ chitosan nanoparticles for oral ovalbumin delivery: Preparation, characterization and intestinal mucosal immunity in mice. Asian J Pharm Sci 2019;14(2):193-203.

45. Kumar A, Dixit CK. Methods for characterization of nanoparticles. Adv Nanomedicine Deliv Ther Nucleic Acids 2017;43:58.

46. Queiroz MF, Melo KRT, Sabry DA, Rocha HAO. Does the Use of Chitosan Contribute to Oxalate Kidney Stone Formation? Mar Drugs 2014;13(1):141-58.

47. Xu Y, Du Y. Effect of molecular structure of chitosan on protein delivery properties of chitosan nanoparticles. Int $\mathrm{J}$ Pharm 2003;250(1):215-26.

48. Qi L, Xu Z. Lead sorption from aqueous solutions on chitosan nanoparticles. Colloids Surfaces A Physicochem Eng Asp 2004;251(1):183-90.

49. Malhotra M, Lane C, Tomaro-Duchesneau C, Saha S, Prakash S. A novel method for synthesizing PEGylated chitosan nanoparticles: strategy, preparation, and in vitro analysis. Int J Nanomedicine 2011;6:485-94.

50. Kandav G, Bhatt DC, Jindal DK. Formulation and evaluation of allopurinol loaded chitosan nanoparticles. Int J Appl Pharm 2019;11(3):49-52.

51. Ferrero F, Periolatto M. Antimicrobial finish of textiles by chitosan UV-curing. J Nanosci Nanotechnol 2012;12(6):480310 .

52. Campos EVR, Proença PLF, Oliveira JL, Melville CC, Della Vechia JF, de Andrade DJ. Chitosan nanoparticles functionalized with $\beta$-cyclodextrin: a promising carrier for botanical pesticides. Sci Rep 2018;8(1):2067.

53. Singla AK, Sharma ML, Dhawan S. Nifedipine loaded chitosan microspheres: characterization of internal structure. Biotech Histochem 2001;76:165-71.

54. Rao VM, Haslam JL, Stella VJ. Controlled and complete release of a model poorly water-soluble drug, prednisolone, from hydroxypropyl methylcellulose matrix tablets using (SBE) $7 \mathrm{~m}-\beta$-cyclodextrin as a solubilizing agent. J Pharm Sci 2001;90(7):807-16. 